\title{
EDITORIAL
}

\section{Diving into the dark kinome: lessons learned from LMTK3}

(c) The Author(s), under exclusive licence to Springer Nature America, Inc. 2021

Cancer Gene Therapy (2022) 29:1077-1079; https://doi.org/10.1038/ s41417-021-00408-3

With $>500$ proteins crucially implicated in a large variety of physiological processes and diseases, the human kinome represents an invaluable source of putative targets with great potential for therapeutic intervention. However, exploring the human kinome as a whole is an ambitious goal still far from being accomplished. Nearly two decades ago, Manning et al. [1] defined a set of 518 protein kinases throughout the human genome. To date, the list of human kinases has substantially expanded counting around 634 kinases, which include both conventional active and 'catalytically dead' kinases [2]. These so-called pseudokinases have gained increased attention over the last years in light of their essential noncatalytic roles in signalling pathways.

Nevertheless, our knowledge of protein targets is confined to a small, yet highly investigated, portion of the kinome, whereas $\sim 25 \%$ of the human kinases are considered to be poorly studied, labelled as 'dark' kinases [2]. For quite a long time, academic research has consistently been quite biased towards kinases that have already been intensively studied. This is also mirrored by the lasting dominance of certain 'favoured' drug targets, especially in oncology. Consequently, a great fraction of the kinome remains without functional annotation, whereas only $~ 8 \%$ of the kinome has so far been effectively targeted for the treatment of cancer [2].

Such a great knowledge disparity, which could also be translated into an opportunity, was addressed by the US National Institutes of Health $(\mathrm{NIH})$ and led to the establishment of the Illuminating the Druggable Genome programme (IDG) [3]. This project was developed with the purpose of encouraging the study of 'dark' proteins that may hold therapeutic relevance and it specifically focused on the understudied members of three large protein families. These included $\mathrm{G}$ protein-coupled receptors, ion channels, and kinases, which offer a rather high number of drug targets. As part of the IDG project, the Kinase Data and Resource Generating Center along with its Dark Kinase Knowledgebase (DKK) and the linked DKK expression browser were developed to specifically enhance our understanding of neglected kinases (Table 1) [4]. This highlights how the appreciation towards the 'dark' kinome continually increases.

Over the last years, a great number of largely uncharacterised kinase targets have emerged from screening studies and genome sequencing efforts. Back in 2011, LMTK3 (lemur tyrosine kinase 3), the core protein of interest in our laboratory, was initially identified as a promising therapeutic target in breast cancer through a kinome siRNA screen that aimed to uncover regulators of the estrogen receptor a (Fig. 1) [5]. Determining and studying protein function can certainly be challenging. Not surprisingly, research decisions can frequently be guided by the existence of relevant knowledge and tools. As a consequence, highly studied kinases are regularly given priority over understudied ones, mainly due to the unpredictable nature of research that may sometimes lead towards the search for a balanced compromise between scientific potential and associated risks. In the case of LMTK3, structural and functional studies aiming to delineate the exact role of LMTK3 in cellular signalling represented a stimulating opportunity that opened a new avenue for investigation. However, the limited relevant literature or insufficient publicly accessible information, lack of antibodies as well as readily available experimental tools presented significant obstacles to overcome.

Ten years later, LMTK3 is an established cancer driver known to act through diverse mechanisms [5-14]. Concisely, the crystal structure of the LMTK3 kinase domain has now been solved and its consensus phosphorylation motif has been determined [12]. In addition, interrogating the signalling networks of LMTK3 revealed a highly versatile functional spectrum for this previously underinvestigated molecular target. Most importantly, a potent and selective small-molecule inhibitor against LMTK3, namely 'C28', has been identified and characterised. Collectively, our work has cast considerable light on LMTK3 providing the academic community with data as well as research tools for the study of LMTK3, while further proving the potential clinical value of 'dark' kinases as relevant cancer targets. Intriguingly, the Clinical Kinase Index [2], a newly developed kinase-prioritisation method aiming to promote the validation and study of understudied kinase targets in cancer, ranked LMTK3 amongst the most clinically relevant kinases across a wide range of cancer types (Table 2). LMTK3, as is the case for other understudied kinases, could serve as a notable example of a druggable target for cancer therapy. Such efforts may potentially lead to the initiation of new drug discovery programmes, while elucidating the role of the numerous, yet unexplored kinases.

Since our present view of cellular signalling is that of an intricate, cooperative and dynamic network, the overriding aim would be to attempt to shed some more light on the 'dark' kinome. In fact, only by widening our knowledge, we can broaden the therapeutic horizon and therefore increase the possibility of successfully addressing currently unmet clinical needs. With the development of a growing number of online resources that can provide a comprehensive overview of the up-to-date knowledge surrounding any kinase, highly relevant and rapidly available information can be used to experimentally assess the potential function of a specific kinase of interest. Leveraging these tools and the multiple improved technologies could help define the functional signature of these underprivileged proteins.

In summary, our work on LMTK3 clearly demonstrates the achievability and benefits of functionally characterising a specific 'dark' kinase, while narrowing the gap between the intensively studied and less-understood kinases. As in the case of LMTK3, several opportunities could arise from such an endeavour, including the further mapping of biological pathways, the unveiling of the role of 'dark' kinases, and the discovery of novel targets. We hope that LMTK3 could serve as an example that may provide other researchers with some of the necessary incentive to take a deep breath and dive into the 'dark' kinome. 
Table 1. The Dark Kinase Knowledgebase (DKK) list of understudied kinases (in alphabetical order) [4].

\begin{tabular}{|c|c|c|c|c|c|}
\hline ADCK1 & CDK20 & ICK & NEK8 & PRKACG & STK32C \\
\hline ADCK2 & CDKL1 & LMTK2 & NEK9 & PRKCQ & STK33 \\
\hline ADCK5 & CDKL2 & LMTK3 & NIM1K & PRPF4B & STK36 \\
\hline ALPK2 & CDKL3 & LRRK1 & NRBP2 & PSKH1 & STK38L \\
\hline ALPK3 & CDKL4 & LTK & NRK & PSKH2 & STK40 \\
\hline BCKDK & CDKL5 & MAP3К10 & NUAK2 & PXK & STKLD1 \\
\hline BRSK1 & CLK3 & МАР3К14 & OBSCN & RIOK1 & TAOK1 \\
\hline CAMK1D & COQ8A & MAP3K21 & PAK5 & RIOK3 & TBCK \\
\hline CAMK1G & COQ8B & MAPK15 & PAK6 & RNASEL & TESK1 \\
\hline CAMKK1 & CSNK1A1L & MAPK4 & PAN3 & RPS6KC1 & TESK2 \\
\hline CAMKK2 & CSNK1G1 & MARK1 & PDIK1L & RPS6KL1 & TLK1 \\
\hline CAMKV & CSNK1G2 & MARK3 & PHKG1 & RSKR & TLK2 \\
\hline CDK11A & DSTYK & MKNK1 & PIP4K2C & SCYL2 & TSSK4 \\
\hline CDK11B & DYRK1B & MKNK2 & PIP5K1A & SCYL3 & TSSK6 \\
\hline CDK12 & DYRK2 & NEK1 & PIP5K1B & SRPK3 & TTBK1 \\
\hline CDK13 & DYRK3 & NEK10 & PIP5K1C & STK17A & TTBK2 \\
\hline CDK14 & DYRK4 & NEK11 & PKMYT1 & STK17B & ULK4 \\
\hline CDK15 & EEF2K & NEK3 & PKN3 & STK19 & VRK2 \\
\hline CDK16 & ERN2 & NEK4 & PNCK & STK3 & VRK3 \\
\hline CDK17 & HIPK1 & NEK5 & POMK & STK31 & WEE2 \\
\hline CDK18 & HIPK3 & NEK6 & PRAG1 & STK32A & WNK2 \\
\hline CDK19 & HIPK4 & NEK7 & PRKACB & STK32B & WNK3 \\
\hline
\end{tabular}

LMTK3, the protein of interest, is shown in bold.
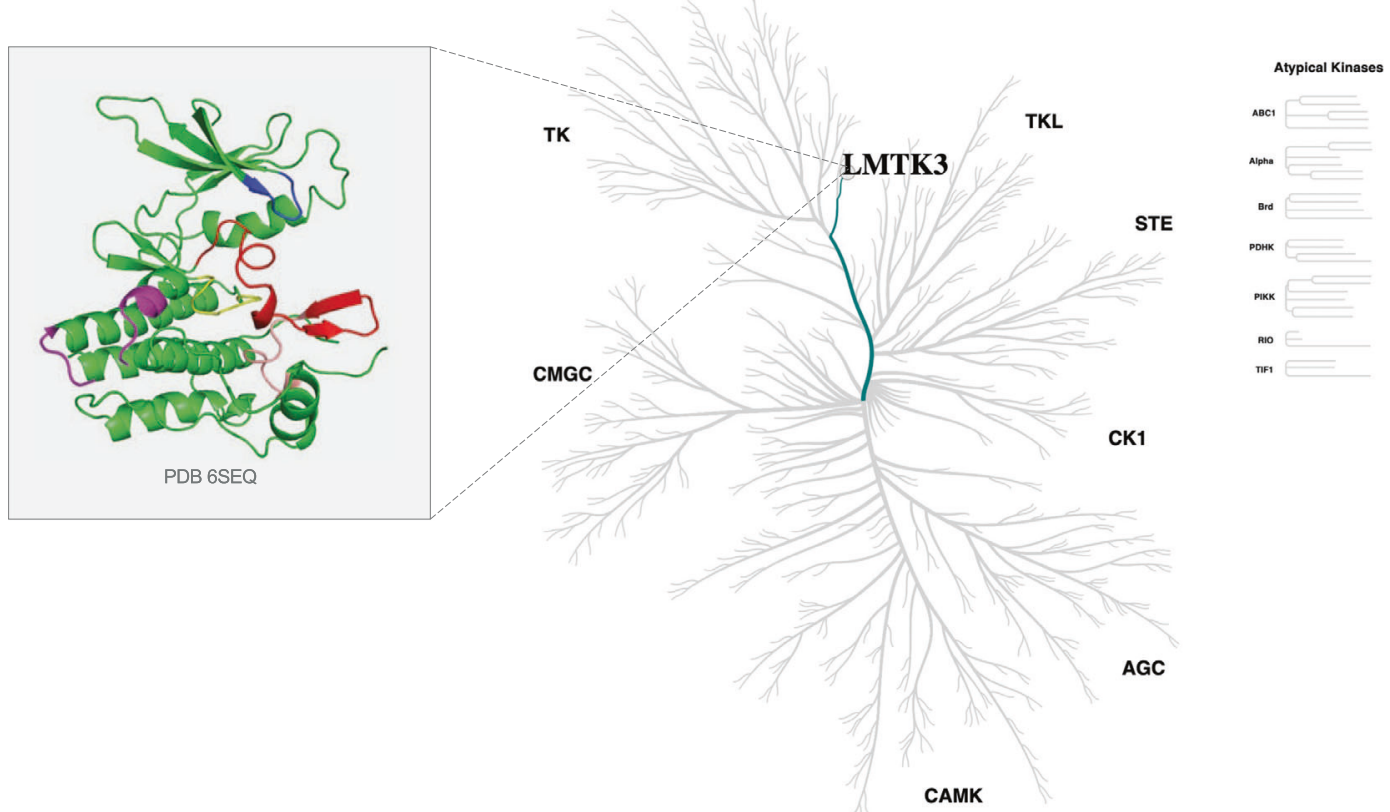

Fig. 1 The location of LMTK3 in the phylogenetic tree of the human kinome [1, 12]. Adapted from the Dark Kinase Knowledgebase (DKK) [4]. 
Table 2. The highest-scoring understudied protein kinases across different cancer types based on the Clinical Kinase Index (CKI) (in alphabetical order) [2].

\begin{tabular}{llll} 
Kinase gene name & Kinase name & Kinase family & Kinase group \\
\hline ADCK5 & Uncharacterised aarF domain-containing protein kinase 5 & ABC1 family & Atypical group \\
\hline BRSK1 & Serine/threonine-protein kinase BRSK1 & CAMKL family & CAMK group \\
\hline DCLK3 & Serine/threonine-protein kinase DCLK3 & DCAMKL family & CAMK group \\
\hline ERN2 & Serine/threonine-protein kinase/endoribonuclease IRE2 & IRE family & Other group \\
\hline LMTK3 & Serine/threonine-protein kinase LMTK3 & LMR family & TK group \\
\hline PKMYT1 & Membrane-associated tyrosine- and threonine-specific cdc2-inhibitory kinase & WEE family & Other group
\end{tabular}

LMTK3 (lemur tyrosine kinase 3) as well as ADCK5 (uncharacterised aarF domain-containing protein kinase 5), BRSK1 (brain-selective kinase 1), DCLK3 (doublecortin-like kinase 3), ERN2 (endoplasmic reticulum-to-nucleus signalling 2), and PKMYT1 (membrane-associated tyrosine- and threonine-specific cdc2inhibitory kinase) potentially represent attractive and clinically relevant targets [2].

LMTK3, the protein of interest, is shown in bold.

Viviana Vella ${ }^{1}$, Georgios Giamas (D) ${ }^{1}$ and Angeliki Ditsiou (iD ${ }^{1 凶}$ ${ }^{1}$ Department of Biochemistry and Biomedicine, School of Life Sciences, University of Sussex, JMS Building, Falmer, Brighton BN1

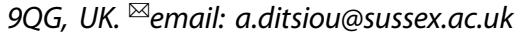

\section{REFERENCES}

1. Manning G, Whyte DB, Martinez R, Hunter T, Sudarsanam S. The protein kinase complement of the human genome. Science 2002;298:1912-34.

2. Essegian D, Khurana R, Stathias V, Schurer SC. The clinical kinase index: a method to prioritize understudied kinases as drug targets for the treatment of cancer. Cell Rep Med. 2020;1:100128.

3. Rodgers G, Austin C, Anderson J, Pawlyk A, Colvis C, Margolis R, et al. Glimmers in illuminating the druggable genome. Nat Rev Drug Discov. 2018;17:301-2.

4. Berginski ME, Moret N, Liu C, Goldfarb D, Sorger PK, Gomez SM. The Dark Kinase Knowledgebase: an online compendium of knowledge and experimental results of understudied kinases. Nucleic Acids Res. 2021;49:D529-D35.

5. Giamas G, Filipovic A, Jacob J, Messier W, Zhang H, Yang D, et al. Kinome screening for regulators of the estrogen receptor identifies LMTK3 as a new therapeutic target in breast cancer. Nat Med. 2011;17:715-9.

6. Stebbing J, Filipovic A, Ellis IO, Green AR, D'Silva TR, Lenz HJ, et al. LMTK3 expression in breast cancer: association with tumor phenotype and clinical outcome. Breast Cancer Res Treat. 2012;132:537-44.

7. Stebbing J, Filipovic A, Lit LC, Blighe K, Grothey A, Xu Y, et al. LMTK3 is implicated in endocrine resistance via multiple signaling pathways. Oncogene 2013;32:3371-80.

8. Xu Y, Zhang H, Lit LC, Grothey A, Athanasiadou M, Kiritsi M, et al. The kinase LMTK3 promotes invasion in breast cancer through GRB2-mediated induction of integrin $\beta_{1}$. Sci Signal. 2014;7:ra58.

9. Xu Y, Zhang H, Nguyen VT, Angelopoulos N, Nunes J, Reid A, et al. LMTK3 represses tumor suppressor-like genes through chromatin remodeling in breast cancer. Cell Rep. 2015;12:837-49.
10. Jacob J, Favicchio R, Karimian N, Mehrabi M, Harding V, Castellano L, et al. LMTK3 escapes tumour suppressor miRNAs via sequestration of DDX5. Cancer Lett. 2016;372:137-46.

11. Stebbing J, Shah K, Lit LC, Gagliano T, Ditsiou A, Wang T, et al. LMTK3 confers chemo-resistance in breast cancer. Oncogene 2018;37:3113-30.

12. Ditsiou A, Cilibrasi C, Simigdala N, Papakyriakou A, Milton-Harris L, Vella V, et al. The structure-function relationship of oncogenic LMTK3. Sci Adv. 2020;6: eabc3099.

13. Cilibrasi C, Ditsiou A, Papakyriakou A, Mavridis G, Eravci M, Stebbing J, et al. LMTK3 inhibition affects microtubule stability. Mol Cancer. 2021;20:53.

14. Ditsiou A, Gagliano T, Samuels M, Vella V, Tolias C, Giamas G. The multifaceted role of lemur tyrosine kinase 3 in health and disease. Open Biol. 2021;11:210218.

\section{AUTHOR CONTRIBUTIONS}

All authors contributed to the writing and editing of the manuscript.

\section{COMPETING INTERESTS}

Georgios Giamas is editor-in-chief of Cancer Gene Therapy and founder/chief scientific officer of Stingray Bio. Angeliki Ditsiou is an editorial-board member of Cancer Gene Therapy.

\section{ADDITIONAL INFORMATION}

Correspondence and requests for materials should be addressed to Angeliki Ditsiou.

Reprints and permission information is available at http://www.nature.com/ reprints

Publisher's note Springer Nature remains neutral with regard to jurisdictional claims in published maps and institutional affiliations. 